\title{
A INVESTIGAÇÃO DAS VISÕES DE PROFESSORES FORMADOS E EM FORMAÇÃO ACERCA DAS INTERAÇÕES ENTRE CIÊNCIA, TECNOLOGIA E SOCIEDADE
}

\author{
Amanda Berk ${ }^{1}$, Roberta da Matta ${ }^{2}$ e Álvaro Chrispino ${ }^{3}$ \\ ${ }^{1,3}$ Centro Federal de Educação Tecnológica - CEFET, Programa de Pós-Graduação em Ciência, Tecnologia e Educação - \\ PPCTE, Rio de Janeiro, RJ. ${ }^{2}$ Universidade do Estado do Rio de Janeiro - UERJ/FFP, São Gonçalo, RJ. E-mail: \\ berk.amanda@yahoo.com.br..Agência de fomento: CAPES
}

\section{RESUMO}

Os conceitos referentes à Ciência, Tecnologia e Sociedade (CTS) estão emergindo e consolidandose diante sua força e relevância no cotidiano da população. O presente artigo visa colaborar com o Projeto de Investigação Iberoamericano em Avaliação de Atitudes Relacionadas com a Ciência, a Tecnologia e a Sociedade (PIERCTS) investigando através de sua metodologia de aplicação de questionários, as crenças de docentes do CEFET/RJ e graduandos acerca das interações e relações CTS. Os resultados revelaram índices atitudinais aquém do esperado por parte dos indivíduos apontando o déficit de formação desses professores e futuros professores acerca da temática CTS. Sendo assim recomenda-se a reforma dos currículos de formação de licenciandos e novas iniciativas de formação continuada para capacitá-los ás práticas pedagógicas sob a ótica CTS.

Palavras-chave: Perspectiva CTS, formação docente, práticas pedagógicas.

\section{THE INVESTIGATION OF THE VIEWS OF TRAINED TEACHERS AND IN EDUCATION ABOUT THE INTERACTIONS BETWEEN SCIENCE, TECHNOLOGY AND SOCIETY}

\begin{abstract}
The concepts related to Science, Technology and Society (STS) are emerging and consolidating itself on its strength and relevance in daily life of the population. This article aims to collaborate with the Iberoamerican Research Project Attitudes Assessment related to Science, Technology and Society (PIERCTS) investigating through its questionnaires application methodology, the beliefs of teachers of CEFET / RJ and graduates about CTS interactions and relationships. The results revealed attitudinal indexes fall short by individuals pointing the training deficit of these teachers and future teachers about the theme CTS. Therefore it is recommended to reform undergraduates training curricula and new continuing education initiatives to enable them ace pedagogical practices from the perspective CTS.
\end{abstract}

Keywords: CTS perspective, teacher training, pedagogical practices. 


\section{INTRODUÇÃO}

Com o avanço da ciência e da tecnologia na sociedade torna-se cada vez mais relevante a discussão sobre suas características, definições e desdobramentos. $\mathrm{O}$ ambiente escolar representa a base científica de formação do indivíduo, que deve através desse contato, refletir sobre o impacto da ciência e da tecnologia no cotidiano assim como os rumos e investimentos conferidos a essas áreas de pesquisa e pensamento. Entretanto, o espaço e carga horária, destinados a essa formação científica são extremamente reduzidos, comprometendo a possibilidade de compreensão ampla e crítica das questões de Ciência, Tecnologia e Sociedade (CTS).

A inclusão dos conceitos relativos à CTS nos currículos de ensino de ciências é conflituosa devido a seu caráter complexo e inovador (VASQUEZ et al., 2008). Contudo, Vaz, Fagundes e Pinheiro (2009) defendem legitimidade do conhecimento científico como verdadeiro para a descoberta de leis e fenômenos amparada pelos conhecimentos proporcionados pela tecnologia para o benefício da sociedade e, portanto a intrínseca relação entre esses aspectos.

Hoje, o currículo acadêmico no âmbito do ensino fundamental e médio está focado em conteúdos que são transmitidos com foco na avaliação com objetivo de resultados de aprovação seja no modelo seriado ou no ingresso das universidades através do vestibular. Há uma linha de pensamento teórico que defende a transformação desse modelo introduzindo o foco na construção do conhecimento, principalmente desenvolvendo o senso crítico e a participação dos discentes. Segundo Auler e Delizoicov (2006) p. 338 as "compreensões de professores sobre interações entre Ciência-Tecnologia-Sociedade (CTS) têm sido apontadas como um dos pontos de estrangulamento, emperrando, muitas vezes, a contemplação do enfoque CTS no processo educacional".

No ambiente escolar, a determinação para a discussão dos conteúdos considerados científicos, geralmente é encontrado no currículo de disciplinas como Ciências e Biologia. A responsabilidade desses profissionais, portanto é grande assim como a exigência de sua formação que determina a capacidade que esse profissional terá de elaborar e articular as informações e conteúdos científicos. Auler e Delizoicov (1999) apontam que a formação disciplinar é um dos desafios para que os docentes consigam realizar seu planejamento didático com o enfoque CTS que preferencialmente seja interdisciplinar.

A formação do professor é fundamental para que o mesmo seja capaz de alcançar as concepções de CTS. Firme e Amaral (2008) investigaram em sua pesquisa que as concepções dos docentes muitas vezes não condizem com o esperado para que seja realizada a mediação de uma discussão crítica assim como a adoção de abordagens CTS em sala de aula. Dessa forma, o 
processo formativo torna-se determinante para que o professor abrace o aspecto CTS em sua prática pedagógica cotidiana.

A cultura CTS é a maneira incorporada dos conceitos que englobam tudo que é representado por CTS. Soligo, Maciel e Guazzelli (2012) argumentam sobre a relevância do ensino sob a perspectiva CTS para viabilizar a incorporação da cultura citada, e afirma inclusive que há uma predisposição e interesse por parte dos alunos para que haja essa abordagem.

A dimensão e abrangência da tríade CTS, muitas vezes não se configura clara para a população. Seus aspectos sociais, políticos e filosóficos por vezes permanecem ocultos aos olhos da maioria. Vaz, Fagundes e Pinheiro (2009) em seu trabalho, discorrem sobre essa multiplicidade de dimensões encontradas no âmbito CTS.

Para a compreensão da temática em questão é basilar o entendimento sobre as interações CTS uma vez que é preciso obter uma visão sobre as influências mútuas e a interdependência que consistem essas relações. Miranda e Freitas (2014) asseguram que a compreensão das interações CTS encontra-se no alicerce da alfabetização científica de docentes e discentes.

O presente trabalho surge do anseio de investigar a diferença entre a visão sobre as interações CTS de professores em exercício no CEFET/RJ e dois grupos de estudantes de graduação dos cursos: Pedagogia e Ciências Biológicas. A hipótese levantada é que os professores terão uma visão mais próxima da realidade assim como os graduandos de Ciências Biológicas apresentaram um discurso mais próximo do adequado a respeito dessas relações e interações CTS.

\section{METODOLOGIA}

A metodologia em questão utiliza o instrumento estipulado através do Projeto de Investigação Iberoamericano em Avaliação de Atitudes Relacionadas com a Ciência, a Tecnologia e a Sociedade (PIERCTS) que consiste em um questionário para investigar as crenças de indivíduos acerca de diversos aspectos CTS. A mesma é considerada qualitativa, pois busca avaliar índices e opiniões atitudinais dos sujeitos que sofreram a aplicação do questionário.

O Questionário de Opiniões sobre a Ciência, a Tecnologia e a Sociedade (COCTS) possui 100 perguntas que compõe diversas questões a respeito da natureza, filosofia e sociologia da Ciência (VÁSQUEZ et al., 2010). As indagações possuem diversas alternativas para as quais o indivíduo deve atribuir uma numeração entre 1 e 9 de acordo com o grau de concordância do participante sobre a afirmativa proposta, conforme observamos na tabela 1 (ANTONIOLI et al., 2012). Esse modelo busca a precisão das respostas e uma análise mais confiabilidade e profundidade nas análises (ANTONIOLI et al., 2011). 
Tabela 1. Numeração disponível aos participantes para atribuírem valores diante de seu índice de concordância

\begin{tabular}{|r|r|r|r|r|r|r|r|r|r|r|}
\hline \multicolumn{4}{|c|}{ DESACORDO } & Indeciso & \multicolumn{4}{r|}{ OUTROS } \\
\hline Total & Alto & Médio & Baixo & & Baixo & Médio & Alto & Total & Não a entendo & Não sei \\
\hline $\mathbf{1}$ & $\mathbf{2}$ & $\mathbf{3}$ & $\mathbf{4}$ & $\mathbf{5}$ & $\mathbf{6}$ & $\mathbf{7}$ & $\mathbf{8}$ & $\mathbf{9}$ & $\mathbf{E}$ & $\mathbf{S}$ \\
\hline
\end{tabular}

Na elaboração do COCTS, 16 juízes considerados especialistas de diferentes formações e atuações científicas, classificaram as alternativas de cada questão entre ingênuas, plausíveis e adequadas (VASQUEZ et al., 2008). Para aferir a proximidade entre a resposta dos participantes e o consenso definido pelos juízes peritos, foi determinado um índice atitudinal variando entre 1 e 1, sendo o mais próximo do acordo dos juízes os números próximos a 1 e os mais distantes e discordantes do considerado apropriado e esperado pelos especialistas.

A aplicação do questionário na pesquisa realizada no CEFET/RJ conta com 30 questões do COCTS subdivididas em dois formulários (1 e 2). A questão escolhida para aplicação foi a 30111 do formulário 1 e pode ser observada na figura 1.

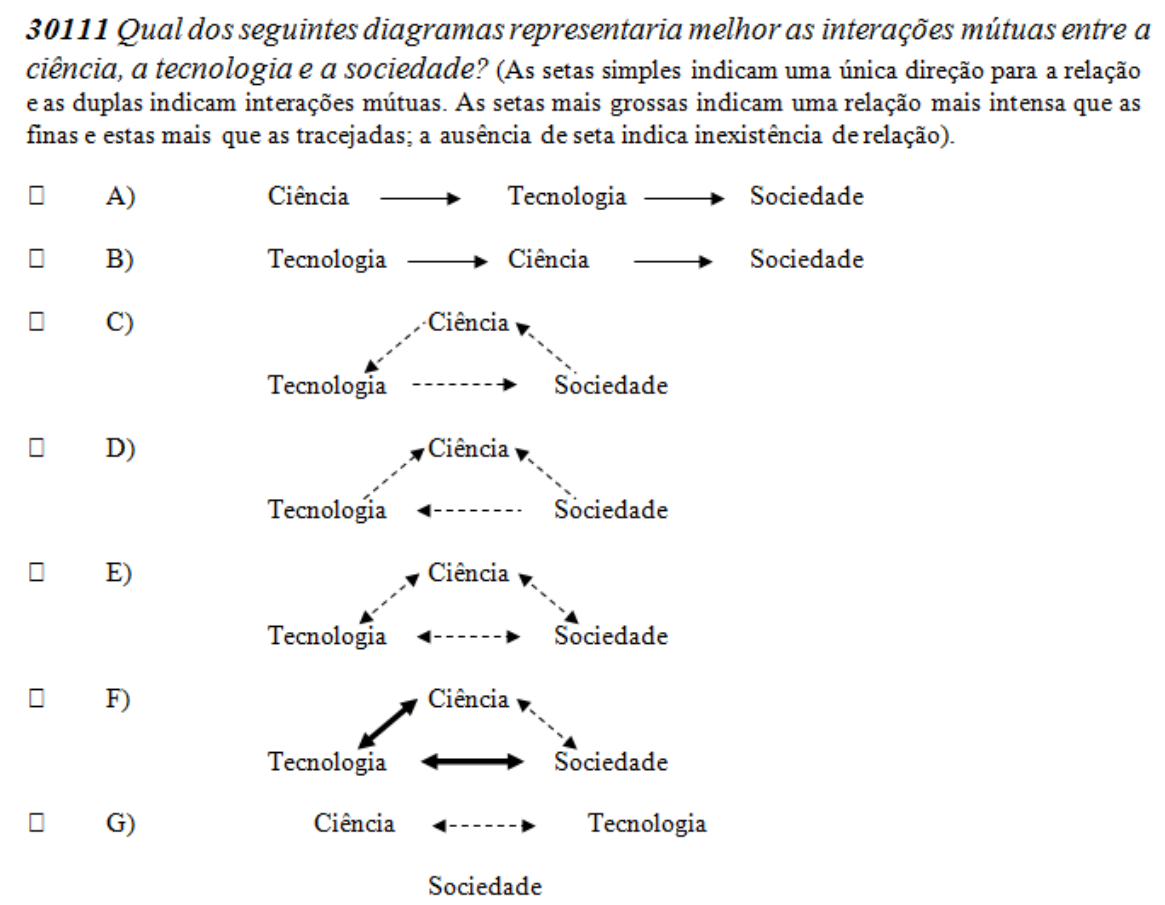

Figura 1. Questão elegida para ser investigada na concepção de docentes e graduandos

Foram escolhidos dois grupos focais para que fosse comparada a percepção desses indivíduos acerca das interações CTS. O primeiro grupo pertence a professores atuantes na instituição de ensino CEFET/RJ. O segundo grupo é composto por duas turmas de graduação de fim de curso com aproximadamente 10 alunos em cada uma dos cursos de Pedagogia e Ciências 
Biológicas da Faculdade de Formação de Professores (FFP) da Universidade do Estado do Rio de Janeiro (UERJ).

\section{RESULTADOS}

De acordo com a definição dos juízes para a questão escolhida (30111), as alternativas A, B, C, D e G são classificadas como ingênuas e as alternativas E e F são adequadas, portanto essa questão não apresenta escolhas plausíveis. Quanto maior o índice de concordância e numerações mais altas atribuídas às opções adequadas melhor a situação dos participantes acerca da compreensão em relação às interações CTS. As numerações mais próximas de 1 são consideradas mais elevadas, já as que possuem decimais que aproximam-se do -1 estão distante do esperado de acordo com as proposições dos juízes.

Na figura 2 observamos os resultados da pesquisa referente à aplicação da questão 30111, realizada com docentes da instituição CEFET/RJ, onde a questão do questionário PIERCTS foi aplicada em um número aproximado de 100 professores. Cerca de 70\% dos professores participantes são da área de humanas e os demais 30\% são da área de Ciências.

Analisando a figura 2 nota-se que as alternativas consideradas como adequadas, no caso da questão 30111 são as representadas pela letra " $E$ " e "F", apresentam uma pontuação baixa quanto aos índices de concordância dos professores submetidos à pesquisa. Isso indica uma baixa consciência desses indivíduos frente às reais interações CTS e suas dimensões.

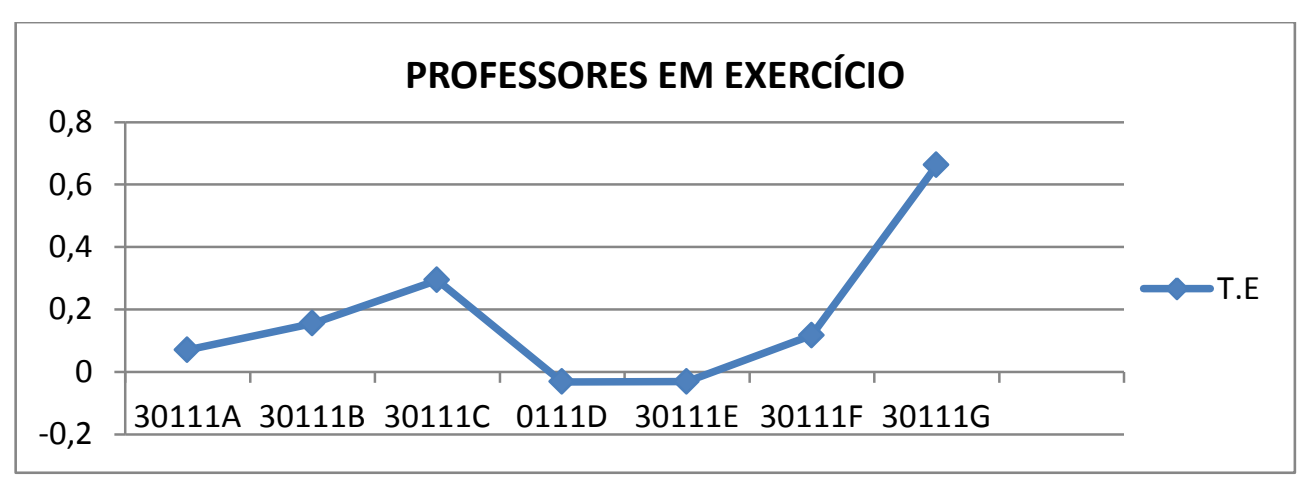

Figura 2. Pontuação dos professores acerca de sua visão sobre as interações CTS.

$\mathrm{Na}$ figura 3 podemos perceber a ondulação da referida pontuação de acordo com as respostas dos graduandos em cada alternativa da questão. Entre os estudantes de pedagogia e ciências biológicas não foi verificada uma diferença significativa em sua percepção acerca das interações CTS. Inclusive a disparidade que foi observada, refutou a hipótese de que graduandos em final de curso com formação voltada para as ciências teriam uma visão mais próxima da 
considerada adequada do que os estudantes de um curso de humanas. O desempenho demonstrado pelos licenciandos em Pedagogia revelou-se mais satisfatório do que os índices apresentados pelos licenciandos de Ciências Biológicas. O resultado obtido pode indicar que durante a formação dos licenciandos de Pedagogia há uma maior discussão acerca dos temas CTS possibilitando aos mesmos uma visão mais crítica sobre a temática.

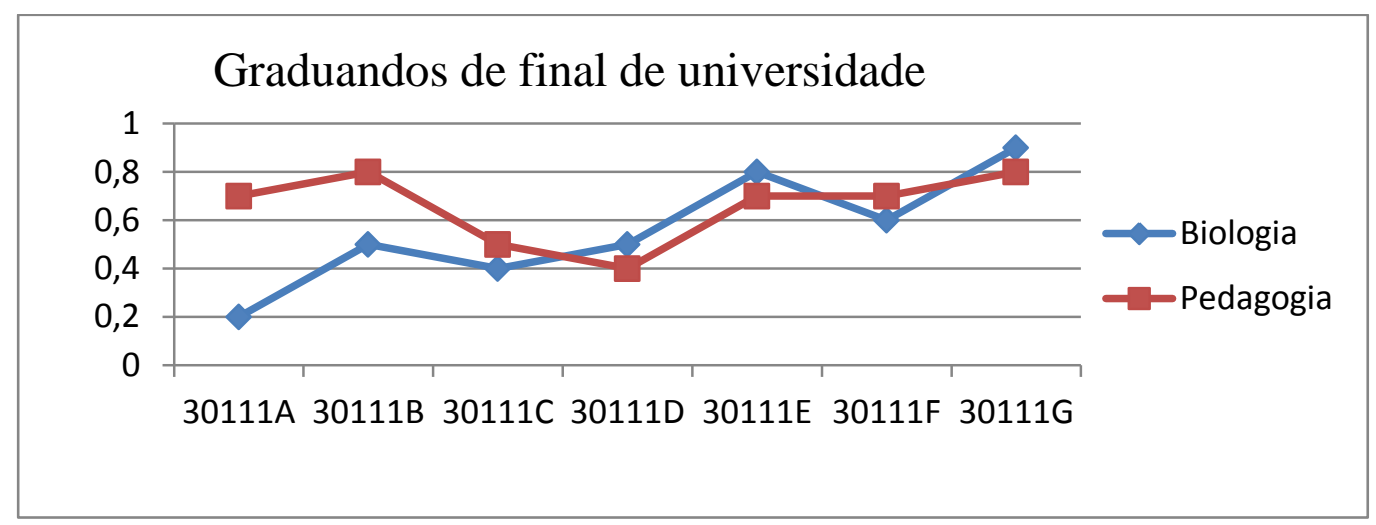

Figura 3. Pontuação de graduandos acerca das interações CTS

Foi investigada a visão dos docentes em exercício parte do corpo docente do CEFET assim como dos graduandos. Com relação à alternativa A podemos verificar a disparidade acentuada entre os três grupos uma vez que os graduandos de pedagogia obtiveram índice de 0,7 em suas respostas, os de Biologia 0,2 e os docentes próximos à zero.

Os resultados apresentados na afirmativa B são similares a A, pois só há a alteração de ordem entre a Ciência e Tecnologia sobrepondo, portanto a tecnologia sobre a ciência e interferindo no seu desenvolvimento. Os valores atitudinais encontrados foram 0,8 para Pedagogia, 0,5 para Biologia e 0,2 para os professores.

A alternativa C representa uma relação fraca diante da linha tracejada presente e os resultados obtidos foram próximos a 0,4 entre os graduandos e 0,3 para os professores. São médias baixas para uma afirmativa que deveria ser nitidamente descartada diante da profunda inter-relação existente entre os componentes de CTS. A frase D expõe diagrama similar à alternativa $C$ somente a direção da interação afirmando que sociedade e tecnologia convergem para ciência apresenta pontuação baixa com os professores próximos a zero e uma superação sutil dos graduandos de Biologia em relação a graduandos de Pedagogia.

As opções $E$ e $F$ que explicitam a relação mais estreita, mútua e intensa entre ciência, tecnologia e sociedade e deveriam apresentar pontuações mais elevadas por parte das respostas dos participantes realmente obtiveram uma numeração mais significativa permeando nos 
graduandos entre 06 e 0,8. Contudo os professores em exercício apresentaram uma pontuação baixa (entre zero e 0,2 ) não identificando essas afirmativas como as adequadas.

O item G que novamente desassocia a sociedade das interações CTS articulando a interação superficial e mútua apenas entre ciência e tecnologia, obteve o maior índice por parte dos docentes acima de 0,6 como também por parte dos graduandos com índices entre 0,8 e 0,9. Indicando que esse modelo apresentando não representa aceitação dos indivíduos.

\section{DISCUSSÕES}

A discrepância observada na alternativa A pode ser analisada pela visão errônea de desenvolvimento linear onde os indivíduos que concordam possuem a crença de que a Ciência possui uma relação de influência quanto à tecnologia e a evolução da tecnologia causa impacto na sociedade. Retomando Miranda e Freitas (2014) não se pode compreender CTS sem a visão apropriada das interações CTS.

Outro aspecto importante de se ressaltar acerca das alternativas A e B é a ausência de relação da sociedade em relação tanto à ciência quanto à tecnologia, dessa forma permanecendo inerte sem possibilidade de interferência o que determina uma distância que compromete o envolvimento dos indivíduos nas decisões e direcionamentos científico tecnológicos. Esse resultado compromete a assimilação da cultura CTS como é proposto por Soligo, Maciel e Guazzelli (2012), tanto por parte dos licenciandos como futuramente por seus alunos em sua prática docente.

A crença de que essas afirmativas podem ser consideradas aceitáveis apontam que os indivíduos não se sentem capazes de participar do processo construtivo da ciência e da tecnologia apesar de acreditarem que essas questões influenciam na sociedade (AULER; DELIZOICOV, 2006). O fato dos graduandos de Pedagogia terem obtido uma visão mais próxima da esperada indica que sua formação apresenta mais elementos questionadores e que inserem a sociedade em todo processo de condução de diversas áreas percebendo que sim a sociedade possui papel ativo nos quesitos referentes à ciência e a tecnologia.

Os índices baixos demonstrados pelos docentes do CEFET/RJ percorrendo os valores de 0,2 ou zero em sua maioria de afirmações corroboram com o argumento de Firme e Amaral (2008) que determinam a falta de embasamento dos docentes para a condução de discussões críticas em CTS. A limitação exposta por Auler e Delizoicov (1999) é explicitada diante desse resultado que aponta essa formação falha. 


\section{CONCLUSÕES}

Diante da similaridade dos resultados obtidos pode-se observar a fragilidade da formação acadêmica dos estudantes de Ciências Biológicas no quesito científico. Essa constatação aponta a necessidade de introduzir o ensino de conteúdos científicos mais contundentes na formação desses licenciandos que serão os futuros formadores na área científica na sociedade.

As concepções de graduandos do curso de Pedagogia estar mais próximas dos índices atitudinais desejados representa uma defasagem na formação de graduando em Biologia assim como a crença apresentada pelos professores em exercício que estão atuando diretamente na formação de novos indivíduos obtendo como sua responsabilidade a disseminação dos conhecimentos pertinentes à CTS.

A discussão acerca das interações CTS, assim como todos os conteúdos relativos à temática devem se inserir no âmbito escolar desde suas bases e há a necessidade de inclusão desse conhecimento como forma de formação continuada para os docentes em exercício e com urgência na formação universitária dos futuros magistrados.

\section{REFERÊNCIAS}

ANTONIOLI, P. M., CHRISPINO, A., VÁZQUEZ, A. \&MANASSERO, M. A., (2012). Avaliação das atitudes das duas culturas em relação à aprendizagem da ciência. Revista iberoamericana de educación. N. 58, pp. 151-166.

AULER, D.; DELIZOICOV, D. Visões de professores sobre as interações entre ciênciatecnologiasociedade (CTS). In: MOREIRA, M. A.; OSTERMANN, F. (Orgs.). Atas do II Encontro Nacional de Pesquisa em Ensino de Ciências. São Paulo: SBF, 1999.

AULER, D.; DELIZOICOV, D. Ciência-Tecnologia-Sociedade: relações estabelecidas por professores de ciências. Revista electrónica de Enseñanza de las Ciencias, v. 5, n. 2, 2006.

FIRME, R. N.; AMARAL, E. M. R. Concepções de professores de química sobre ciência, tecnologia, sociedade e suas inter-relações: um estudo preliminar para o desenvolvimento de abordagens CTS em sala de aula. Ciência \& Educação, Bauru, v. 14, n. 2, p. 251-269, 2008. http://dx.doi.org/10.1590/s1516-73132008000200005

MIRANDA, M. E.; FREITAS, D. Um olhar CTS sobre as concepções de professores de ciências através do questionário VOSTS. In: Congresso Ibero Americano de Ciência, Tecnologia, Inovação e Educação. Anais... Buenos Aires, dez. 2014.

SOLIGO, MG.; MACIEL, MD.; GUAZZELLI, IRB. IMPORTÂNCIA DE SE LEVAR EM CONSIDERAÇÃO AS INTERAÇÕES DE ALUNOS ADOLESCENTES COM A MÍDIA TELEVISIVA E REDES SOCIAIS PARA UMA PROPOSTA DE ACT DE CUNHO CTS. Revista de Ensino de Ciências e Matemática, v. 3, n. 3, p. 269281, 2012. 
VASQUEZ, A. A.; MANASSERO, M. A.; ACEVEDO, J. A. D.; ROMERO, P. A. Consensos sobre a Natureza da Ciência: A Ciência e a Tecnologia na Sociedade. Química Nova na escola, n. 27, fev., 2008.

VÁZQUEZ, Á.; MANASSERO, M. A. e TALAVERA, M. Actitudes y creencias sobre naturaleza de la ciencia y la tecnología em uma muestra representativa de jóvenes estudiantes, Revista Electrónica de Enseñanza de las Ciencias, 9( 2): 333-352, 2010.

VAZ, C. R.; FAGUNDES, A. B. e PINHEIRO, N. A. M. O surgimento da ciência, tecnologia e sociedade (CTS) na educação: uma revisão. Anais do I Simpósio Nacional de Ensino de Ciência e Tecnologia, Curitiba, 2009. 